\title{
How Zonal Flow Affects Trapped-Electron-Driven Turbulence in Tokamak Plasmas
}

\author{
Haotian Chen $\oplus^{1,2}$ and Liu Chen $\oplus^{3,4,5, *}$ \\ ${ }^{1}$ Institute of Space Science and Technology, Nanchang University, Nanchang 330031, China \\ ${ }^{2}$ Department of Atomic, Molecular and Nuclear Physics, University of Seville, Seville 41012, Spain \\ ${ }^{3}$ Institute for Fusion Theory and Simulation and Department of Physics, Zhejiang University, Hangzhou 310027, China \\ ${ }^{4}$ Department of Physics and Astronomy, University of California, Irvine, California 92697, USA \\ ${ }^{5}$ Center for Nonlinear Plasma Science and C.R. ENEA Frascati-C.P. 65, 00044 Frascati, Italy
}

(Received 11 August 2021; revised 21 November 2021; accepted 17 December 2021; published 12 January 2022)

\begin{abstract}
The role of self-generated zonal flows in the collisionless trapped-electron-mode (CTEM) turbulence is a long-standing open issue in tokamak plasmas. Here, we show, for the first time, that the zonal flow excitation in the CTEM turbulence is formally isomorphic to that in the ion temperature gradient turbulence. Trapped electrons contribute implicitly only via linear dynamics. Theoretical analyses further suggest that, for short wavelength CTEMs, the zonal flow excitation is weak and, more importantly, not an effective saturation mechanism. Corresponding controlling parameters are also identified theoretically. These findings not only offer a plausible explanation for previous seemingly contradictory simulation results, but can also facilitate controlling the CTEM instability and transport with experimentally accessible parameters.
\end{abstract}

DOI: 10.1103/PhysRevLett.128.025003

Zonal flows (ZFs) are azimuthally symmetric sheared flows spontaneously excited by small scale fluctuations. They are common in both nature and laboratory, and are crucial for the self-regulation of turbulence and transport. In tokamak plasmas, the drift wave-zonal flow paradigm, as one of major achievements of modern plasma turbulence theory, has been established after intensive studies of the ion temperature gradient (ITG) turbulence (see [1] and references therein). At present it has been widely accepted that ZF-induced energy transfer from the unstable mode to stable modes provides the primary saturation mechanism for ITG turbulence [2,3]. However, noting that fusion power increases as the square of density and fusion produced alpha particles mainly heat electrons, the collisionless trapped-electron-mode (CTEM) turbulence may show more pronounced effects in future burning plasmas such as ITER [4]. That is, in addition to playing a key role in particle transport, CTEM also significantly contributes to electron heat transport in ITER scenarios with dominant electron heating and/or with comparable electron and ion heating. Although it has long been recognized that the CTEM turbulence is of theoretical and practical interests, the significance of ZF in regulating the CTEM turbulence, nevertheless, is still being actively debated. Numerical

Published by the American Physical Society under the terms of the Creative Commons Attribution 4.0 International license. Further distribution of this work must maintain attribution to the author(s) and the published article's title, journal citation, and DOI. studies have shown that the role of ZF in CTEM turbulence is parameter sensitive, with different controlling parameters identified from different simulations [5-14]. Specifically, the ZF excitation is empirically found to be sensitive either to the ratio between electron and ion temperatures $(\tau)$, magnetic shear $(s)$, and electron temperature gradient scale length $\left(r_{t e}\right)$ [6-9]; or to $\eta_{e}$ (the ratio between gradients of the density and electron temperature) only [13]. It is conjectured that the importance of ZF may be connected with the linear stability of CTEM [13], but the underlying physics mechanism is as yet unknown. The need for a clear physical picture of CTEM ZF interplay is thus the main motivation for this work.

In this Letter, we employ the nonlinear gyrokinetic theory [15] and demonstrate analytically that the $\mathrm{ZF}$ excitation in CTEM turbulence is formally isomorphic to that in ITG turbulence. Interestingly, although the turbulence is driven by trapped electrons, the nonlinear CTEM ZF interplay is governed by ions and circulating electrons. Trapped electrons, in contrast, only enter implicitly through linear physics. Therefore, linear CTEM properties play a unique role in determining the importance of $\mathrm{ZF}$. Theoretical analysis elucidates that ZFs are important in saturating the long wavelength CTEM turbulence, consistent with simulation results $[5,7-9,11-14]$. For the short wavelength CTEM turbulence (to be defined later), however, ZF excitation is weak and, more significantly, not an effective saturation channel. Linear short wavelength CTEMs are thus revisited analytically. It is found that the short wavelength CTEM instability without ZF scattering channel is essentially of two types. One is kinetically 
excited via toroidal precessional resonance. In this case, the instability threshold depends on the aspect ratio between major and minor radii, the temperature ratio, magnetic shear, and electron temperature gradient; consistent with previous numerical simulation observations in Refs. [6-9]. The other case is a fluidlike interchange-driven instability set by $\eta_{e}$ in the steep density gradient regime, explaining thereby the simulation results in Ref. [13]. We note that while this work analyzes CTEMs in tokamaks, the theoretical approach presented here could conceivably be applicable toward clarifying the role of ZF in other plasma turbulence; e.g., shear Alfvén waves excited by either energetic [16] or thermal [17] particles, where waveparticle interactions of different particle species occur on distinctively separated spatial scales.

We consider an axisymmetric, low- $\beta$ (the ratio of kinetic to magnetic pressure), large aspect-ratio $\left(\epsilon=r / R_{0} \ll 1\right)$ tokamak with the major radius $R_{0}$, and the radial ( $r$, distance from the magnetic axis), poloidal $(\theta)$, and toroidal $(\zeta)$ fieldaligned coordinates. The electrostatic fluctuation is taken to be coherent and consists of the pump CTEM $A_{0},\left(\omega_{0}, \boldsymbol{k}_{0}\right)$, upper and lower sideband CTEMs $A_{ \pm},\left(\omega_{ \pm}, \boldsymbol{k}_{ \pm} \equiv \boldsymbol{k}_{0} \pm k_{z} \hat{e}_{r}\right)$, and a zonal mode $A_{z},\left(\omega_{z}, k_{z} \hat{e}_{r}\right)$. For CTEMs, since the instability drive peaks on the outboard midplane, we can assume eigenmodes are mainly formed within $|\theta| \leq \pi$, and adopt the strongly ballooning representation [18]

$$
\left\{\phi_{k}, \delta h_{k}\right\}=A_{k} e^{-i n q \theta_{k}} e^{-i n(\zeta-q \theta)}\left\{\Phi_{k}(\theta), \delta H_{k}(\theta)\right\} .
$$

Here, $q(r)$ stands for the safety factor, $A_{k}$ denotes the amplitude, and $n q^{\prime} \theta_{k}$ is the radial envelope wave number. For simplicity, we take $\theta_{k}=0$ for the pump and $k_{z}=n q^{\prime} \theta_{z}$ for the zonal mode, hence the radial envelope wave number of upper (lower) sideband becomes $\pm k_{z}$. The nonlinear equation for the electrostatic potential $\phi_{k}$ is the quasineutrality condition:

$$
(1+\tau) \phi_{k}+\left\langle\delta h_{e, k}\right\rangle_{v}-\left\langle J_{k} \delta h_{i, k}\right\rangle_{v}=0,
$$

where $\tau=T_{e} / T_{i}$ is the temperature ratio between electron and ion, $\langle\cdots\rangle_{v}$ denotes integration in velocity space, and $J_{\boldsymbol{k}}=J_{0}\left(k_{\perp} \rho_{i} v_{\perp}\right)$ is a Bessel function accounting for the finite Larmor radius effect of ions, with $\rho_{i}$ being the thermal ion Larmor radius and velocity being normalized to the thermal velocity. The nonadiabatic response $\delta h_{j, k}$, meanwhile, obeys the nonlinear gyrokinetic equation [15]

$$
\begin{aligned}
\mathcal{L}_{j, \boldsymbol{k}} \delta h_{j, \boldsymbol{k}}-\left(\omega+\omega_{* j}^{t}\right) F_{0} J_{\boldsymbol{k}} \frac{q_{j} \phi_{\boldsymbol{k}}}{T_{j}} \\
=\frac{i c}{B} \sum_{\boldsymbol{k}_{2}=\boldsymbol{k}-\boldsymbol{k}_{1}}\left\{\left[J_{\boldsymbol{k}_{1}} \phi_{\boldsymbol{k}_{1}}, \delta h_{j, \boldsymbol{k}_{2}}^{*}\right]+\left[J_{\boldsymbol{k}_{2}} \boldsymbol{\phi}_{\boldsymbol{k}_{2}}^{*}, \delta h_{j, \boldsymbol{k}_{1}}\right]\right\} .
\end{aligned}
$$

Here, $j=i, e$ denotes particle species, the charge $q_{i}=-q_{e}=e$, and $\mathcal{L}_{j, k}=\omega+\omega_{t}+\omega_{d j}$ is the phase-space propagator in toroidal geometry, in which $\omega_{t}=i\left(v_{\|} / q R_{0}\right) \partial_{\theta}$ is the transit frequency, and $\omega_{d j}=2 k_{\theta} v_{\|}^{2} G /\left(\omega_{c j} R_{0}\right)$ is the curvature drift model for magnetic drift frequency [19], with $G \equiv \cos \theta+s\left(\theta-\theta_{k}\right) \sin \theta, k_{\theta}=n q / r$, and $\omega_{c j}=q_{j} B /\left(c_{j}\right)$. $\omega_{* j}^{t}=\omega_{* j}\left[1+\eta_{j}\left(v^{2}-3 / 2\right)\right]$ is the diamagnetic frequency, where $\omega_{* j}=k_{\theta} c T_{j} /\left(q_{j} B r_{n}\right), \eta_{j}=r_{n} / r_{t j}$, and $r_{n}$ and $r_{t j}$ are, respectively, the density and temperature scale lengths. For clarity of the physics presentation, we set $\eta_{i}=0$ to inhibit ion driven modes. $F_{0}$ is Maxwellian. We have also defined the Poisson bracket $[f, g]=i\left(k_{\theta, f} f \partial_{r} g-k_{\theta, g} g \partial_{r} f\right)$ for the $\boldsymbol{E} \times \boldsymbol{B}$ nonlinearity on the right-hand side, with $k_{\theta, f}\left(k_{\theta, g}\right)$ denoting the poloidal wave number $k_{\theta}$ of $f(g)$.

For trapped electrons, Eq. (3) can be further reduced to the nonlinear bounce kinetic equation [20]:

$$
\begin{aligned}
\mathcal{L}_{t e, \boldsymbol{k}} \delta H_{t e, \boldsymbol{k}}+\left(\omega+\omega_{* e}^{t}\right) F_{0} \frac{e \bar{\Phi}_{\boldsymbol{k}}}{T_{e}} \\
=\frac{i c}{B} \sum_{\boldsymbol{k}=\boldsymbol{k}_{1}-\boldsymbol{k}_{2}}\left\{\left[\bar{\Phi}_{\boldsymbol{k}_{1}}, \delta H_{t e, \boldsymbol{k}_{2}}^{*}\right]+\left[\bar{\Phi}_{\boldsymbol{k}_{2}}^{*}, \delta H_{t e, \boldsymbol{k}_{1}}\right]\right\},
\end{aligned}
$$

where $\overline{(\cdots)}=\left[\oint(\cdots) d \theta / v_{\|}\right] / \oint d \theta / v_{\|}$denotes bounce or transit averaging [21]. The propagator $\mathcal{L}_{t e, k}=\omega-\bar{\omega}_{d e}$, with the precessional frequency $\bar{\omega}_{d e}=\left|\omega_{* e}\right| \epsilon_{n} v^{2} H$, $\epsilon_{n}=r_{n} / R_{0}$, and $H \simeq 0.83 s+0.41$ for $s \sim 1[22,24]$ typically employed in simulations [5-14].

Although linear CTEMs have been extensively explored in the literature since 1970s [24-26], it is nevertheless crucial to discuss the linear properties useful for nonlinear analysis. For this, the eigenmode equation is derived from linearized kinetic equations as [27]

$$
\begin{aligned}
\hat{D} \Phi \equiv & (1+\tau) \Phi-\left\langle J_{0} \mathcal{L}_{i}^{-1}\left[\left(\omega+\omega_{* i}^{t}\right) \tau F_{0} J_{0} \Phi\right]\right\rangle_{v} \\
& -\left\langle\mathcal{L}_{t e}^{-1}\left[\left(\omega+\omega_{* e}^{t}\right) F_{0} \bar{\Phi}\right]\right\rangle_{v}=0 .
\end{aligned}
$$

Equation (5) distinguishes two types of CTEMs. The long wavelength mode $\left[k_{\perp}^{2} \rho_{i}^{2} \sim \mathcal{O}(\epsilon)\right]$ comes from a balance between the adiabatic term $(1+\tau)$ and the nonadiabatic ion response (the second term), which requires that the nonadiabatic trapped-electron response is subdominant; while the short wavelength mode $\left[k_{\perp}^{2} \rho_{i}^{2} \sim \mathcal{O}\left(\epsilon^{-1}\right)\right]$ is defined here as the result of a balance between the adiabatic term and nonadiabatic trapped-electron response, implying a subdominant nonadiabatic ion contribution.

As to the excitation of ZF by CTEM turbulence, we may follow the analyses of Refs. [2,28,29]. The particle response to ZF can be solved from Eq. (3), by employing the separation in time scales between the low frequency zonal flow and the bounce (or transit) frequency of guidingcenter motion [28]. The quasineutrality condition of zonal mode then becomes

$$
\left(\partial_{t}+\gamma_{z}\right) \chi_{i} A_{z}=k_{z} \rho_{i}\left(A_{+} A_{0}^{*} \beta_{z,+}-A_{0} A_{-}^{*} \beta_{z,-}^{*}\right) .
$$


Here, frequencies and amplitudes are normalized to $\left|\omega_{* e}\right|$ and $\left(T_{e} / e\right)\left(\rho_{i} / r_{n}\right)$, respectively. $\gamma_{z}$ is the collisional $\mathrm{ZF}$ damping rate. $\chi_{i}=\tau\left(1-\left\langle\left\langle F_{0} J_{z} e^{i Q} \overline{e^{-i Q}} J_{z}\right\rangle\right\rangle_{v, s}\right)$ quantifies the classical and neoclassical polarization of ions [28], with $Q=k_{z} v_{\|} q /\left(\omega_{c i} \epsilon\right)$ accounting for the ion finite drift orbit width effect, and $\langle\cdots\rangle_{s}$ being the average over magnetic flux surface. The nonlinear coupling coefficient is given by [29] $\beta_{z, \pm}=\left\langle\left\langle J_{k_{z}} e^{i Q} \overline{e^{-i Q}\left(J_{\boldsymbol{k}_{ \pm}} \Phi_{\boldsymbol{k}_{ \pm}} \delta H_{i, \boldsymbol{k}_{0}}^{*}\right)}\right\rangle\right\rangle_{v, s}-$ $\left\langle\left\langle\Phi_{\boldsymbol{k}_{ \pm}} J_{\boldsymbol{k}_{0}} \delta H_{i, \boldsymbol{k}_{0}}^{*}\right\rangle\right\rangle_{v, s}-\left\langle\left\langle J_{k_{z}} e^{i Q} \overline{e^{-i Q}\left(J_{\boldsymbol{k}_{0}} \Phi_{\boldsymbol{k}_{0}}^{*} \delta H_{i, \boldsymbol{k}_{ \pm}}\right)}\right\rangle\right\rangle_{v, s}+$ $\left\langle\left\langle\Phi_{\boldsymbol{k}_{0}}^{*} J_{\boldsymbol{k}_{ \pm}} \delta H_{i, \boldsymbol{k}_{ \pm}}\right\rangle\right\rangle_{v, s^{*}}$. Consistently, with $\theta_{k}=0$ for the pump mode, one can show that $\beta_{z,+}=\beta_{z,-} \equiv \beta_{z}$ for sidebands of the same branch by virtue of the up-down symmetry [25]: $\Phi_{k_{+}}=\Phi_{k_{-}}$and $\omega_{k_{+}}=\omega_{k_{-}}$. Since the characteristic CTEM frequency is much smaller than the electron transit frequency, the circulating electron response to CTEMs is adiabatic. The corresponding electron contribution to $\beta_{z, \pm}$ is thus linearly proportional to $\left\langle\left\langle\overline{\left[\phi_{\boldsymbol{k}_{ \pm}}, \delta h_{t e, \boldsymbol{k}_{0}}^{*}\right]}\right\rangle\right\rangle_{v, s}-\left\langle\left\langle\left[\boldsymbol{\phi}_{\boldsymbol{k}_{ \pm}}, \delta h_{t e, \boldsymbol{k}_{0}}^{*}\right]\right\rangle\right\rangle_{v, s}+$ $\left\langle\left\langle\overline{\left[\phi_{\boldsymbol{k}_{0}}^{*}, \delta h_{t e, \boldsymbol{k}_{ \pm}}\right]}\right\rangle\right\rangle_{v, s}-\left\langle\left\langle\left[\phi_{\boldsymbol{k}_{0}}^{*}, \delta h_{t e, \boldsymbol{k}_{ \pm}}\right]\right\rangle\right\rangle_{v, s}$. Meanwhile, recalling that the bounce kinetic limit holds for trapped electrons, it is possible to show that $\left\langle\left\langle\overline{\left[\phi_{\boldsymbol{k}_{1}}, \delta h_{t e, \boldsymbol{k}_{2}}^{*}\right]}\right\rangle\right\rangle_{v, s}=$ $\left\langle\left\langle\left[\bar{\Phi}_{\boldsymbol{k}_{1}}, \delta H_{t e, \boldsymbol{k}_{2}}^{*}\right]\right\rangle\right\rangle_{v, s}=\left\langle\left\langle\left[\phi_{\boldsymbol{k}_{1}}, \delta h_{t e, \boldsymbol{k}_{2}}^{*}\right]\right\rangle\right\rangle_{v, s}[20]$. As a consequence, trapped electrons do not contribute to $\beta_{z, \pm}$. The nonlinear terms recover the well-known CharneyHasegawa-Mima nonlinearity in long wavelength limit [30]. It is, therefore, clear that the ZF equation is only set by ion dynamics, as that in the familiar ITG turbulence. Specifically, assuming $k_{\perp 0}^{2} \rho_{i}^{2} \sim k_{z}^{2} \rho_{i}^{2} \ll 1$, it is not hard to show that Eq. (6) is mathematically identical to the $\mathrm{ZF}$ equation in $[2,29]$.

For CTEM sidebands, the nonlinear coupling to the pump and ZF can be straightforwardly derived by closely following Refs. [2,29], yielding

$$
\left(\partial_{t}+i \Delta_{+}-\gamma_{+}\right) A_{+}=-k_{z} \rho_{i} \alpha_{+} A_{0} A_{z} / \omega_{+} \partial_{\omega} D_{+},
$$

and

$$
\left(\partial_{t}+i \Delta_{-}-\gamma_{-}\right) A_{-}=k_{z} \rho_{i} \alpha_{-} A_{0} A_{z}^{*} / \omega_{-} \partial_{\omega} D_{-} .
$$

Here, $\Delta_{ \pm}=\omega_{ \pm}-\omega_{0}$ is frequency mismatch, $\gamma_{ \pm}$is sideband linear damping rate, and $D_{k}$ represents the real part of linear dispersion function $\left\langle\Phi_{k}^{*} \hat{D} \Phi_{k}\right\rangle_{s}$. The mode coupling $\alpha_{ \pm} \equiv$ $\alpha_{e \pm}+\tau \alpha_{i \pm}$ here originates from both the Navier-Stokes type $\boldsymbol{E} \times \boldsymbol{B}$ nonlinearity $\alpha_{e \pm}=(1-\sqrt{2 \epsilon})\left\langle\Phi_{\boldsymbol{k}_{ \pm}}^{*} \Phi_{\boldsymbol{k}_{0}}\right\rangle_{s}$ via circulating electrons; and the generalized Reynolds stress $\alpha_{i \pm}=$ $\left\langle\Phi_{\boldsymbol{k}_{ \pm}}^{*}\left(1-\left\langle J_{\boldsymbol{k}_{ \pm}} J_{\boldsymbol{k}_{0}} J_{k_{z}} e^{i Q} \overline{e^{-i Q}} F_{0}\right\rangle_{v}\right) \Phi_{\boldsymbol{k}_{0}}\right\rangle_{s}$ due to ions. While the former dominates in the long wavelength limit, they have comparable magnitudes for short wavelength modes.

Similarly, the pump CTEM equation is readily obtained as

$$
\left(\partial_{t}-\gamma_{0}\right) A_{0}=k_{z} \rho_{i}\left[\alpha_{+} A_{+} A_{z}^{*}-\alpha_{-} A_{-} A_{z}\right] / \omega_{0} \partial_{\omega} D_{0} .
$$

From Eqs. (7)-(9), the nonlinear terms are formally independent of trapped electrons, and possess the conservation law of the wave energy of CTEM plasmons $E_{k}=\omega_{k} \partial_{\omega} D_{k}\left|A_{k}\right|^{2}$ :

$$
\left(\partial_{t}-2 \gamma_{0}\right) E_{0}=\left(2 \gamma_{+}-\partial_{t}\right) E_{+}+\left(2 \gamma_{-}-\partial_{t}\right) E_{-} .
$$

The weak turbulence theory introduced so far applies for scatterings to the linearly stable domain in both dominant [2] and subdominant [3] branches. For a direct comparison with previous ITG results [2,29], we now restrict consideration to the mode coupling in the same branch and analyze the modulational instability with a constant pump. Letting $\Delta_{s}=\Delta_{ \pm}, \gamma_{s}=\gamma_{ \pm}, D=D_{0} \simeq D_{ \pm}$, and $\alpha=\alpha_{ \pm}$, Eqs. (6)-(8) yield the desired nonlinear dispersion relation

$$
\frac{\gamma_{m}^{2}\left(\gamma_{s}-\Gamma_{z}\right)\left|A_{0}\right|^{2} \operatorname{Re}\left(\beta_{z}\right)}{\left(\Gamma_{z}+\gamma_{z}\right)\left[\left(\Gamma_{z}-\gamma_{s}\right)^{2}+\Delta_{s}^{2}\right]}=1
$$

with $\Gamma_{z} \equiv \partial_{t}$ and $\gamma_{m}^{2} \equiv 2 k_{z}^{2} \rho_{i}^{2} \alpha /\left(\chi_{i} \omega_{0} \partial_{\omega} D\right)$. Equation (11) admits a threshold amplitude of the pump,

$$
\left|A_{0, c}\right|^{2}=\gamma_{z} \gamma_{s}\left(1+\Delta_{s}^{2} / \gamma_{s}^{2}\right) / \gamma_{m}^{2} \operatorname{Re}\left(\beta_{z}\right),
$$

above which the $\mathrm{ZF}$ growth rate can be expressed, for $\Gamma_{z}>\left|\gamma_{s}\right|, \gamma_{z}$, as

$$
\begin{aligned}
2 \Gamma_{z}= & \sqrt{\left(\gamma_{s}+\gamma_{z}\right)^{2}-4\left[\operatorname{Re}\left(\beta_{z}\right) \gamma_{m}^{2}\left|A_{0}\right|^{2}-\gamma_{z} \Delta_{s}^{2} / \gamma_{s}\right]} \\
& +\left(\gamma_{s}-\gamma_{z}\right) .
\end{aligned}
$$

Similarities with the ITG driven zonal flow [2] become evident. Therefore, the CTEM turbulence and ITG turbulence are formally isomorphic in $\mathrm{ZF}$ excitation. The nonlinear dynamics is governed by ions and circulating electrons. The trapped-electron contribution, on the other hand, only enters implicitly through linear physics. For this reason, linear CTEM properties are expected to play a determinant role in the ZF excitation. This explains the empirical observation from gyrokinetic simulations that the role of ZF appears to be connected to linear CTEM properties [13].

Equations (12) and (13) also elucidate that the nonlinear coupling coefficient $\beta_{z}$ is crucial for ZF excitation. Noting that the spectrum of long wavelength CTEMs has a similar scale to that of ITGs [24], it is straightforward to show that $\alpha \simeq 1-\sqrt{2 \epsilon}$ and $\omega_{0} \simeq 1 /\left(1+\tau b_{i 0}\right)$ with $b_{i 0}=k_{\perp 0}^{2} \rho_{i}^{2} / 2 \ll 1$, the coefficient $\beta_{z}$ can then be easily computed as [2]

$$
\beta_{z} \simeq-\left(1 / \omega_{0}+\tau\right) k_{z}^{2} \rho_{i}^{2} / 2 .
$$

Substituting Eq. (14) into Eqs. (12) and (13), one readily concludes that both the threshold $\left|A_{0, c}\right|^{2}$ and the ZF growth 
rate $\Gamma_{z}$ in long wavelength CTEM turbulence are comparable to those in ITG turbulence [2]. That is, the CTEM ZF interplay occurs on a time scale similar to that of ITG turbulence, and, thus, is expected to play important roles in saturating the long wavelength CTEM turbulence, as observed by gyrokinetic simulations [5,7-9,11-14]. The generic parameter dependence of ZF effects, however, is beyond the intended scope of the current study, since an adequate modeling of long wavelength CTEM spectrum is still lacking.

Conversely, the nonlinear coupling coefficients for short wavelength modes $\left(b_{i 0} \gg 1\right)$ can be evaluated, after some straightforward algebra, as $\alpha \simeq 1+\tau$ and

$\beta_{z} \simeq-\left\langle\frac{\tau\left(1+\tau \omega_{0}\right) k_{z}^{2} \rho_{i}^{2}}{2 \sqrt{\pi} k_{\perp 0}^{3} \rho_{i}^{3}} \frac{\left|\Phi_{k_{0}}\right|^{2}}{4 \epsilon_{n} G} \frac{Z\left(\sqrt{-\tau \omega_{0} / 4 \epsilon_{n} G}\right)}{\sqrt{-\tau \omega_{0} / 4 \epsilon_{n} G}}\right\rangle_{s}$,

which is $\mathcal{O}\left(\pi^{-1 / 2} k_{\perp 0}^{-3} \rho_{i}^{-3}\right)$ smaller than that in Eq. (14). In deriving Eq. (15), we have neglected the transit frequency in the ion propagator $\mathcal{L}_{i, k}$, and assumed $k_{z}^{2} \rho_{i}^{2} \ll 1$, since the short wavelength $\mathrm{ZF}$ has a strong polarization shielding effect $\chi_{i} \simeq \tau$ and is more difficult to excite. $Z$ is the usual plasma dispersion function. A comparison with the long wavelength CTEM case reveals that the value of $\left|A_{0, c}\right|^{2}$ is enhanced by at least $\mathcal{O}\left(\pi^{1 / 2} k_{\perp 0}^{3} \rho_{i}^{3}\right)$ for a fixed $k_{z}^{2} \rho_{i}^{2}$. Physically, this phenomenon is due to the fact that, in the short wavelength limit, the fast ion gyromotion can average out fluctuations and thus decouple $\mathrm{ZF}$ from CTEMs. Therefore, the ZF excitation becomes much weaker in the short wavelength CTEM turbulence, consistent, again, with numerical simulation results [13].

Now the key issue is to determine the stability of short wavelength CTEMs. For this purpose, we explore the eigenmode equation (5) in the adiabatic ion limit [22,25]

$$
(1+\tau) \Phi+\sqrt{2 \epsilon} T \int_{\sin ^{2} \frac{\theta}{2}}^{1} \frac{d \kappa^{2} \bar{\Phi}}{\sqrt{\kappa^{2}-\sin ^{2} \frac{\theta}{2}}}=0,
$$

where the bounce averaged potential is $\bar{\Phi}=$ $\int_{-\theta_{b}}^{+\theta_{b}} \Phi d \vartheta /\left[4 K(\kappa) \sqrt{\kappa^{2}-\sin ^{2}(\vartheta / 2)}\right]$, with $K$ the complete elliptic integral of first kind and $\theta_{b}=2 \sin ^{-1} \kappa$ the turning point; and $T=\left\{2 T_{1}\left[\omega\left(1-\eta_{e} / \epsilon_{n} H\right)-1\right]+\eta_{e}\left(3 T_{1}-1\right)\right\} / \epsilon_{n} H$, with $T_{1}=1+\sqrt{\omega / \epsilon_{n} H} Z\left(\sqrt{\omega / \epsilon_{n} H}\right)$ accounting for the precessional resonance. Solving Eq. (16) numerically yields the most unstable mode $\Phi_{k_{0}} \simeq(1+\cos \theta) / 3 \pi$ and an algebraic dispersion relation

$$
\lambda_{0}(1+\tau)+\sqrt{2 \epsilon} T=0,
$$

where $\lambda_{0} \simeq 1.2$, Assuming the linear marginal stability is achieved at a critical real frequency $\omega_{\mathrm{cr}}$, the threshold $\eta_{e}$ can then be derived by setting the real and imaginary parts of
Eq. (17) to zero. We find, for modes propagating in electron diamagnetic direction $\left(\omega_{\mathrm{cr}}>0\right)$,

$$
\eta_{e, c 1}=2 \epsilon_{n} / 3 \epsilon_{n c},
$$

with $\epsilon_{n c} \equiv 2 \sqrt{2 \epsilon} /\left[3 H \lambda_{0}(1+\tau)\right]$, and the critical real frequency $\omega_{\mathrm{cr} 1}=3\left(\epsilon_{n}-\epsilon_{n c}\right) H /\left(2-3 \epsilon_{n c} H\right)$. Moreover, according to Eq. (5), self-consistency with the ordering $\left|\left\langle J_{0} \mathcal{L}_{i}^{-1}\left[\left(\omega+\omega_{* i}^{t}\right) \tau F_{0} J_{0}\right]\right\rangle_{v}\right| \ll(1+\tau)$ requires that, in the $\tau \omega \gg \epsilon_{n}$ limit, $\omega \gg \Gamma_{0} /\left(1+\tau-\tau \Gamma_{0}\right)$, with $\Gamma_{0}=I_{0}\left(b_{i}\right) e^{-b_{i}}$ and $I_{0}$ a modified Bessel function. Including the higherorder nonadiabatic ion term, the linear growth rate near marginal stability can be obtained iteratively from Eq. (5) as

$$
\gamma_{0}=\frac{\operatorname{Im}\left(T_{1}^{-1}\right) \epsilon_{n}^{2} H^{2}}{\epsilon_{n} H-\eta_{e, c 1}}\left[\frac{\eta_{e}-\eta_{e, c 1}}{2 \epsilon_{n} H}+\frac{\left(1+\tau \omega_{\mathrm{cr} 1}\right) \Gamma_{0}}{2 \sqrt{2 \epsilon} \lambda_{0}^{-1} \omega_{\mathrm{cr} 1}}\right] .
$$

Therefore, the mode is kinetically excited via the finite toroidal precessional resonance, and the nonadiabatic ion correction is destabilizing.

The expression for $\omega_{\text {crl }}$ implies that the positive frequency assumption will break down for small $\epsilon_{n}<\epsilon_{n c}$. In this case, by expanding Eq. (17) around $\left|\omega_{\text {cr }} /\left(\epsilon_{n} H\right)\right| \ll 1$, one finds

$$
\begin{aligned}
0= & 6 \epsilon_{n c}\left(2-4 \eta_{e}+\epsilon_{n} H\right) \xi^{2}-3 \sqrt{\pi} \epsilon_{n c}\left(2-3 \eta_{e}\right) \xi-2 \epsilon_{n} \\
& +6 \epsilon_{n c}\left(1-\eta_{e}\right),
\end{aligned}
$$

with $\xi \equiv \sqrt{-\omega_{\text {cr }} /\left(\epsilon_{n} H\right)}$. Thus, the CTEM considered here is a fluidlike interchange-driven instability [21], with the following marginal stability condition

$$
\begin{aligned}
\eta_{e, c 2}= & \frac{8}{9 \pi-64}\left\{\frac{3 \pi}{4}-6+\left(\frac{4 \epsilon_{n}}{3 \epsilon_{n c}}-\epsilon_{n} H\right)\right. \\
& -\left\{\left[\frac{3 \pi}{4}-6+\left(\frac{4 \epsilon_{n}}{3 \epsilon_{n c}}-\epsilon_{n} H\right)\right]^{2}-\left(\frac{9 \pi}{4}-16\right)\right. \\
& \left.\left.\times\left[\frac{\pi}{4}-2+\left(\frac{4 \epsilon_{n}}{3 \epsilon_{n c}}\left(2+\epsilon_{n} H\right)-\epsilon_{n} H\right)\right]\right\}^{1 / 2}\right\} .
\end{aligned}
$$

The critical real frequency is given by $\xi_{\mathrm{cr} 2}=\sqrt{\pi}\left(3 \eta_{e}-2\right) /$ $\left(16 \eta_{e}-8-4 \epsilon_{n} H\right)$.

From Eq. (18), it is instructive to note that the system exhibits a striking feature that when $\epsilon_{n}>\epsilon_{n c}$, the fundamental threshold in Eq. (18) is a critical temperature gradient rather than an $\eta_{e}$. Consequently, noting the expression of $\epsilon_{n c}$, the marginal stability condition is set by the parameters $\epsilon, \tau$, $r_{t e}$, and $s$, consistent with numerical results [5-12]. On the contrary, when the density profile is sufficiently steep such that $\epsilon_{n}<\epsilon_{n c}$, the interchange-driven mode becomes unstable above a certain critical $\eta_{e, c 2}$. Especially in the $\epsilon_{n} \ll \epsilon_{n c}$ limit, the marginal condition (21) renders $\eta_{e, c 2} \simeq 1.165$ regardless of other parameters. Note that for current 


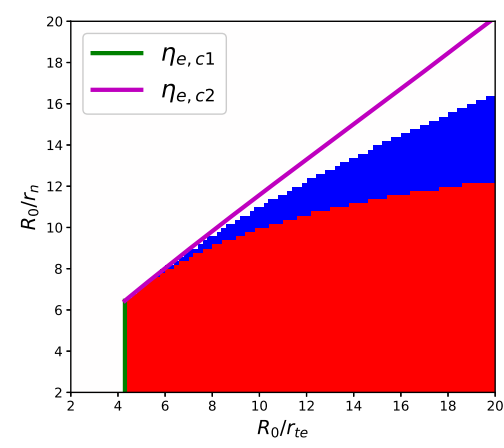

FIG. 1. Stability diagram of short wavelength CTEM, for $\tau=1, \epsilon=0.18$ and $s=0.8$. White is stable, and blue (red) represents unstable modes propagating in the ion (electron) diamagnetic direction.

parameters, the nonuniformity gradient remains weak such that one can ignore global profile variations and subdominant modes in deriving Eq. (17) [22,31].

Confirmation that the adiabatic ion assumption captures the essential physics of short wavelength CTEMs can be obtained by solving Eq. (17) numerically and comparing the results with existing simulations. Figure 1 shows the resultant stability diagram versus the density and temperature gradient scale lengths for parameters in Ref. [13]. Three different regions can be identified: the white region is stable to short wavelength CTEMs, while the blue (red) region is unstable to modes propagating in ion (electron) diamagnetic direction. It also validates the analytical marginal stability conditions. As seen in Fig. 1, the marginal stability is indeed set by a critical $\eta_{e}$ for steep density gradient plasmas with $\epsilon_{n}<\epsilon_{n c}$, whereas it corresponds to a critical $r_{t e} / R_{0}$ for $\epsilon_{n}>\epsilon_{n c}$. Remarkably, these features are in both qualitative and quantitative agreement with simulation results (see Fig. 1 in Ref. [13]). Moreover, as $\eta_{e}$ moves across the threshold from the blue (red) to white region, one would expect that long wavelength modes eventually dominate the dynamics and ZFs become increasingly more important, as observed in simulations [13].

Under the assumption of adiabatic ions, the linear dispersion relation, Eq. (17), is independent of the toroidal mode number. In this scenario, a broad spectrum of short wavelength CTEMs is expected, extending from $\left|k_{\theta} \rho_{i}\right| \gtrsim 1$ to $\left|k_{\theta} \rho_{e}\right| \simeq \mathcal{O}(1)$, until the adiabatic approximation of circulating electrons becomes invalid ultimately. Therefore, once excited, the short wavelength CTEM turbulence characterized by weak ZF excitation will have a broad energy-containing spectrum.

Perhaps the most significant property of short wavelength CTEMs is that they can exist with little stabilization due to zonal flows. More specifically, noting that short wavelength CTEMs arise from the balance between the adiabatic term and the nonadiabatic trapped-electron response, and trapped electrons tend to bounce along magnetic field lines, the eigenmode equation (5) is, in the lowest-order adiabatic ion limit, essentially independent of the radial envelope modulation $\left(\theta_{k}\right)$ effect. Therefore, in contrast to the ITG case, the short wavelength CTEM can be locally excited without a short radial wavelength stable domain in the dominant branch. Meanwhile, owing to the Hermitian nature of Eq. (16), the mode structures of different branches are orthogonal [22]. Thus, besides the aforementioned small $\beta_{z, \pm}$, the coefficients $\alpha_{ \pm}$vanish for the coupling between different branches, and ZFs can barely be excited in this scenario. Given that, as inferred from Eq. (10), the damping of driving mode is due to scatterings to the linearly stable domain in either dominant [2] or subdominant [3] branches, one can conclude that ZF excitation will not be an effective saturation channel for short wavelength CTEMs.

In summary, we have demonstrated the isomorphism between CTEM turbulence and ITG turbulence in zonal flow excitation. The nonlinear CTEM ZF interplay is determined by ions and circulating electrons. Trapped electrons, due to their negligible finite drift orbit width and Larmor radius effects, only enter implicitly via linear physics. The linear properties of CTEM, therefore, play a fundamental role in ZF excitation. While ZF scattering can be important in saturating long wavelength CTEMs, it is found that short wavelength CTEMs can exist with little stabilization due to the zonal flow excitation, and, thereby, renders the conventional drift wave-zonal flow paradigm invalid here. Controlling parameters of the short wavelength CTEM instability are also identified analytically. This new understanding, thus, provides a plausible explanation for the seemingly contradictory features observed in previous simulations. Finally, we remark that current results also carry significant implications to tokamak experiments. In particular, the short wavelength CTEM turbulence could be a candidate to explain the observed insensitivity of electron heat transport to $\boldsymbol{E} \times \boldsymbol{B}$ shearing rate as well as the decoupling of the electron thermal diffusivity and the particle diffusion, due to the ambipolarity [32]. Equations (18) and (21), meanwhile, can be utilized to identify the experimentally accessible parameters and thus control the spatiotemporal scale of CTEM turbulence. It may pave a way for proactively controlling CTEM-driven electron transport and optimizing fusion plasma performance.

We acknowledge discussions with Z. Qiu, F. Zonca, E. Viezzer, and M. Gracia-Munoz. H. T. Chen particularly wants to thank the ongoing and unwavering support of X. K. Fang. This work was supported by National Natural Science Foundation of China under Grant No. 11905097, and the European Unions Horizon 2020 research and innovation programme (No. 805162).

*Corresponding author. liuchen@zju.edu.cn

[1] P. H. Diamond, S-I. Itoh, K. Itoh, and T. S. Hahm, Plasma Phys. Controlled Fusion 47, R35 (2005). 
[2] L. Chen, Z. Lin, and R. White, Phys. Plasmas 7, 3129 (2000).

[3] K. D. Makwana, P. W. Terry, M. J. Pueschel, and D. R. Hatch, Phys. Rev. Lett. 112, 095002 (2014).

[4] M. Shimada et al., Nucl. Fusion 47, S1 (2007).

[5] D. R. Ernst et al., Phys. Plasmas 11, 2637 (2004).

[6] T. Dannert and F. Jenko, Phys. Plasmas 12, 072309 (2005).

[7] R. Waltz and C. Holland, Phys. Plasmas 15, 122503 (2008).

[8] J. Lang, Y. Chen, and S. E. Parker, Phys. Plasmas 14, 082315 (2007).

[9] J. Lang, S. E. Parker, and Y. Chen, Phys. Plasmas 15, 055907 (2008).

[10] S. Jolliet, Ph.D. thesis, Ecole polytechnique federale de Lausanne, 2009.

[11] Y. Xiao and Z. Lin, Phys. Rev. Lett. 103, 085004 (2009).

[12] Y. Xiao, I. Holod, W. Zhang, S. Klasky, and Z. Lin, Phys. Plasmas 17, 022302 (2010).

[13] D. R. Ernst, J. Lang, W. M. Nevins, M. Hoffman, Y. Chen, W. Dorland, and S. Parker, Phys. Plasmas 16, 055906 (2009).

[14] M. Nakata, M. Honda, M. Yoshida, H. Urano, M. Nunami, S. Maeyama, T.-H. Watanabe, and H. Sugama, Nucl. Fusion 56, 086010 (2016).

[15] R. A. Frieman and L. Chen, Phys. Fluids 25, 502 (1982).

[16] L. Chen and F. Zonca, Rev. Mod. Phys. 88, 015008 (2016).

[17] W. W. Heidbrink, M. A. Van Zeeland, M. E. Austin, A. Bierwage, L. Chen, G. J. Choi, P. Lauber, Z. Lin, G. R. McKee, and D. A. Spong, Nucl. Fusion 61, 016029 (2021).
[18] J. W. Connor, R. J. Hastie, and J. B. Taylor, Phys. Rev. Lett. 40, 396 (1978).

[19] P. Terry, W. Anderson, and W. Horton, Nucl. Fusion 22, 487 (1982).

[20] F. Y. Gang and P. H. Diamand, Phys. Fluids B 2, 2976 (1990).

[21] W. Tang, Nucl. Fusion 18, 1089 (1978).

[22] See Supplemental Material at http://link.aps.org/supplemental/ 10.1103/PhysRevLett.128.025003 for a detailed discussion on the eigenmode equation of short wavelength modes, which includes Refs. [21,23-25].

[23] H. T. Chen, J. Comput. Appl. Math. 402, 113796 (2022).

[24] J. Adam, W. Tang, and P. Rutherford, Phys. Fluids 19, 561 (1976).

[25] H. T. Chen and L. Chen, Plasma Phys. Controlled Fusion 60, 055011 (2018).

[26] B. Coppi and G. Rewoldt, Phys. Rev. Lett. 33, 1329 (1974).

[27] H. T. Chen and L. Chen, Nucl. Fusion 59, 074003 (2019).

[28] M. N. Rosenbluth and F. L. Hinton, Phys. Rev. Lett. 80, 724 (1998).

[29] F. Zonca, R. B. White, and L. Chen, Phys. Plasmas 11, 2488 (2004).

[30] A. Hasegawa and K. Mima, Phys. Rev. Lett. 39, 205 (1977).

[31] H. T. Chen and L. Chen, Phys. Plasmas 25, 014502 (2018).

[32] C. C. Petty, M. R. Wade, J. E. Kinsey, D. R. Baker, and T. C. Luce, Phys. Plasmas 9, 128 (2002). 